\title{
Multiplicative functional on elementary fuzzy matrices
}

Thangaraj Beaula ${ }^{1 *}$ and Mallika ${ }^{2}$

\author{
Abstract \\ In this paper some new elementary fuzzy matrices are defined, some of its properties using multiplicative \\ functional are studied. \\ Keywords \\ Fuzzy matrices, elementary fuzzy matrices, multiplicative functional, fuzzy addition and multiplication. \\ AMS Subject Classification \\ 15B15, 15B10, 03E73, 04A72. \\ ${ }^{1}$ P.G and Research Department of Mathematics, TBML College, Porayar, India. \\ ${ }^{2}$ Department of Mathematics, Dharmapuram Adhinam College, Mayiladuthurai, India. \\ ${ }^{*}$ Corresponding author: ${ }^{1}$ edwinbeaula@yahoo.co.in \\ Article History: Received 21 December 2018; Accepted 11 February 2019

\section{Contents}

1 Introduction 45

2

3

Preliminaries .45

Some results of multiplicative functional on elementary fuzzy matrices $. . \ldots \ldots \ldots \ldots \ldots \ldots \ldots \ldots \ldots, 46$

References .47

\section{Introduction}

Fuzzy sets and fuzzy matrices play an important role in scientific development. Fuzzy sets were presented by L.A. Zadeh[12] in 1965, and fuzzy matrices were introduced by Thomason[10] in 1977. Various properties relative to fuzzy set and fuzzy matrix were subsequently studied by many authors, for example,[1]-[3] presented a number of results on the convergence of the power sequence of fuzzy matrices respectively. In 1980, K.H. Kim and F.W. Roush [5] studied the canonical form of an idempotent matrix.

In 1995, Ragab et al.[9] presented some properties of the min-max composition discussed triangular fuzzy matrices. With regard to determinant theory of fuzzy matrix J.B. Kim[5], [6] discussed some properties of determinant theory for fuzzy and Boolean matrices in 1988.M.Z.Ragab and E.G. Emam[8] presented the theory of the determinant and adjoint of a square fuzzy matrix in 1994. R. Hemasinha, N.R. Pal and J.C. Bezdek[2] studied the determinant of a fuzzy matrix with respect to t-norm and co- norms in 1997.

\section{Preliminaries}

Definition 2.1. Let $F[0,1]$ denote the fuzzy algebra over the interval $[0,1]$ with fuzzy addition + and fuzzy multiplication operation $\times$ defined as

$$
a+b=\max \{a, b\} \text { and } a \times b=\min \{a, b\}
$$

for alla, $b \in[0,1]$ and the standard order $\geq$.

Definition 2.2. If $A=\left[a_{i j}\right]$ is a $m \times n$ matrix and every $a_{i j} \in$ $F[0,1]$, the matrix $A$ is said to be a fuzzy matrix.

Definition 2.3. The fuzzy addition and fuzzy multiplication of $m \times n$ fuzzy matrices $A=\left[a_{i j}\right]$ and $B=\left[b_{i j}\right]$ are respectively defined as

$A+B=\left[a_{i j}+b_{i j}\right]=\left[\max \left\{a_{i j}, b_{i j}\right\}\right]$

and

$A \times B=\left[\max \left\{\min \left\{a_{i k}, b_{k j}\right\}, 1 \leq k \leq m\right\}\right]$

Definition 2.4. If $f: F_{n}, n \rightarrow F[0,1]$,

$$
f(A B)=f(A) f(B)
$$

for any $A, B \in F_{n, n}$ then $f$ is said to be multiplicative functional, if a functional

$f: F[0,1] \rightarrow F[0,1]$ satisfies

$f(a b)=f(a) f(b)$ for any $a, b \in F$

Definition 2.5. Let $A=\left(a_{i j}\right)$ be a fuzzy matrix of order $n$ then the matrix $\left(A^{k}\right)$ is obtained from the matrix $A$ by adding $\lambda$ times $i^{\text {th }}$ row(column) to the $k^{\text {th }}$ row (column) of $A$. 
Definition 2.6. Let $A=\left(a_{i j}\right)$ be a fuzzy matrix of order ' $n$ ' then the matrix $\left({ }_{K} A\right)$ is defined as,

$\left.{ }_{K} A\right)={ }_{K} a_{i j}$, where ${ }_{K} a_{i j}=\lambda_{i j}^{a}$, when $K=i$

$=0$, otherwise

Definition 2.7. Let $A=\left(a_{i j}\right)$ be a fuzzy matrix of order $n$ then the relation between $\left(A^{k}\right)$ and $\left.{ }_{K} A\right)$ is obtained by multiplying all the elements of $i^{\text {th }}$ row by a non-zero number $\lambda$.

$A^{K}=A+{ }_{K} A$

\section{Some results of multiplicative functional on elementary fuzzy matrices}

Theorem 3.1. Let $f: F_{n, n} \rightarrow F[0,1]$ be multiplicative linear functional then,

(i) $f\left(A^{K}\right)=f(A)+f\left({ }_{K} A\right)$

(ii) $f\left(A^{K}+B^{K}\right)=f\left(A^{K}\right)+f\left(B^{K}\right)$

(iii) $f\left(A^{K} B^{K}\right)=f\left(\left(A B^{K}\right)+\lambda(\lambda+1) f(A B)\right.$

Proof. (i) By definition,

$A^{k}=\left(a_{i j}^{k}\right)$ where $\left(a_{i j}^{k}\right)=a_{k j}+a_{i j} k=1,2,3, \ldots, n$

$$
\begin{aligned}
\left(A^{k}\right) & =f\left(a_{i j}^{k}\right) \\
& =f\left(a_{k j}+\lambda_{i j}\right) \\
& =f\left(a_{k j}+f\left(\lambda a_{i j}\right)\right. \\
& =f(A)+f\left({ }_{K} A\right)
\end{aligned}
$$

where $_{K} a_{i j}=\lambda_{i j}^{a}$, when $K=1$

$=0$, otherwise

Therefore, $f\left(A^{k}\right)=f(A)+f\left({ }_{K} A\right)$.

(ii)

$$
\begin{aligned}
f\left(A^{k}+B^{k}\right) & =f\left(a_{k j}+\lambda a_{i j}+b_{k j}+\lambda b_{i j}\right) \\
& =f\left(a_{k j}+\lambda a_{i j}\right)+f\left(b_{k j}+\lambda_{i j}\right) \\
& =f\left(A^{k}\right)+f\left(B^{k}\right)
\end{aligned}
$$

(iii)

$$
\begin{aligned}
f\left(A^{k} B^{k}\right) & =f\left(a_{i j}^{k} b_{i j}^{k}\right) \\
f & \left(\sum_{k} a_{i k}+\lambda a_{i k}+b_{k j}+\lambda b_{k j}\right) \\
f & \left(\sum a_{i k} b_{k j}+\lambda \sum a_{i k} b_{k j}+\lambda \sum a_{i k} b_{k j}+\lambda^{2} \sum a_{i k} b_{k j}\right) \\
f & \left\{\sum\left(a_{i k} b_{k j}+\lambda a_{i k} b_{k j}\right)+\left[\lambda \sum a_{i k} b_{k j}(1+\lambda)\right]\right\} \\
f & \left.\sum\left(a_{i k}\right) b_{k j}+\lambda a_{i k} b_{k j}\right)+\lambda(\lambda+1) f\left(\sum a_{i k} b_{k j}\right) \\
f\left(A^{k} B^{k}\right) & =f\left(A B^{k}\right)+\lambda(\lambda+1) f(A B)
\end{aligned}
$$

Theorem 3.2. Let $A=\left(a_{i j}\right)$ be a fuzzy matrix of order ' $n$ ' let $f: F_{n, n} \rightarrow F[0,1]$ be the multiplicative functional and $F_{0}(K)=$ $f\left(A^{k}\right)$ then

(i) $F_{0}(1)=f\left({ }_{1} A\right)+f\left({ }_{K} A\right)$

(ii) $F_{0}\left(k_{1} k_{2}\right)=f\left(A^{k_{1}}\right) f\left(A^{k_{2}}\right)+f\left({ }_{K} A\right)$
Proof. (i) Since,

$$
\begin{aligned}
F_{0}(K) & =f\left(A^{k}\right) \\
& =f\left(a_{i j}^{k}\right) \\
F_{0}(K) & =f\left(a_{k j+\lambda a_{i j}}\right.
\end{aligned}
$$

Take $\mathrm{k}=1$

$$
\begin{aligned}
F_{0}(1) & =f\left(a_{1 j}+\lambda a_{i j}\right) \\
& =f\left(a_{1 j}\right)+f\left(\lambda a_{i j}\right) \\
& =f\left(1^{a}\right)+f\left({ }_{K} A\right) \text { where }{ }_{K} A=\lambda a_{i j}
\end{aligned}
$$

(ii)

$$
\begin{aligned}
F_{0}\left(K_{1} K_{2}\right) & =f\left(A^{K_{1}, K_{2}}\right) \\
& =f\left(a_{i j}^{K_{1} K_{2}}\right) \\
& =f\left(a_{k_{1} k_{2} j}+\lambda a_{i j}\right) \\
& =f\left(a_{k_{1} j}\right) f\left(a_{k_{2} j}+f\left(\lambda a_{i j}\right)\right) \\
F_{0}\left(K_{1} K_{2}\right) & =f\left(A^{k_{1}}\right) f\left(A^{k_{2}}\right)+f\left({ }_{K} A\right) .
\end{aligned}
$$

Theorem 3.3. Let $f: F_{n, n} \rightarrow F[0,1]$ be multiplicative functional and $H_{0}(\lambda)=f\left(A^{K}\right)$ then,

(i). $H_{o}(0)=f(A)$ and

(ii). $H_{o}\left(K_{1}+K_{2}\right)=H_{o}\left(K_{1}\right)+H_{o}\left(K_{2}\right)$

Proof. (i) Since $H_{o}(0)=f\left(A^{k}\right)$

$$
\text { put } \lambda=0
$$$$
=f\left(a_{k j}+\lambda a_{i j}\right)
$$

$$
\begin{aligned}
H_{o}(0) & =f\left(a_{k j}+o . a_{i j}\right) \\
& =f\left(a_{k j}\right) \text { where } k=1,2,3, \text { dots }, n \text { and } j=1,2,3, \text { dots }, n \\
H_{o}(0) & =f(A)
\end{aligned}
$$

(ii)

$$
\begin{aligned}
\left.H_{o}\left(K_{1}+K_{2}\right)\right) & =f\left(A^{k_{1}+k_{2}}\right) \\
& =f\left(a_{\left(k_{1}+k_{2}\right) j}+\lambda\left(a_{k_{1} j}+a_{k_{2} j}\right)\right)+\lambda a_{k_{2} j} \\
& =f\left[\left(a_{k_{1} j+\lambda a_{k_{1} j}}\right)+\left(a_{k_{2} j}+\lambda a_{k_{2} j}\right)\right] \\
& =f\left(a_{k_{1} j}+\lambda a_{k_{1} j}\right)+f\left(a_{k_{2} j}+\lambda a_{k_{2} j}\right) \\
& =f\left(A^{k_{1}}\right)+f\left(A^{k_{2}}\right) \\
& =H_{o}\left(K_{1}\right)+H_{o}\left(K_{2}\right)
\end{aligned}
$$

Theorem 3.4. Let $A=\left(a_{i j}\right)$ be a fuzzy matrix of order $n$ then $A^{\left(k_{1}+k_{2}\right)}\left(\lambda_{1} \lambda_{2}\right)=A^{k_{1} \lambda_{1}}+A^{k_{2} \lambda_{2}}-\lambda_{1}\left(1-\lambda_{2}\right) A-\lambda_{2}\left(1-\lambda_{1}\right) A$.

Proof.

$$
\begin{aligned}
A^{\left(k_{1}+k_{2}\right)}\left(\lambda_{1} \lambda_{2}\right) & =a_{\left(k_{1}+k_{2}\right) j}+\lambda_{1} \lambda_{2} a_{\left(k_{1}+k_{2}\right) j} \\
& =a_{k_{1} j}+a_{k_{2} j}+\lambda_{1} \lambda_{2}\left(a_{k_{1} j}+a_{k_{2} j}\right) \\
& =a_{k_{1} j}+a_{k_{2} j}+\lambda_{1} a_{k_{1} j}+\lambda_{2} a_{k_{2} j}-\lambda_{1} a_{k_{1} j} \\
& -\lambda_{2} a_{k_{2} j}+\lambda_{1} \lambda_{2}\left(a_{k_{1} j}+a_{k_{2} j}\right) \\
& =\left(a_{k_{1} j}+\lambda_{1} a_{k_{1} j}\right)+\left(a_{k_{2} j}+\lambda_{2} a_{k_{2} j}\right)
\end{aligned}
$$




$$
\begin{aligned}
& -\lambda_{1} a_{k_{1} j}\left(1-\lambda_{2}\right)-\lambda_{2}\left(1-\lambda_{1}\right) a_{k_{2} j} \\
& =A^{k_{1} \lambda_{1}}+A^{k_{2} \lambda_{2}}-\lambda_{1}\left(1-\lambda_{2}\right) A-\lambda_{2}\left(1-\lambda_{1}\right) A .
\end{aligned}
$$

Theorem 3.5. Let $A=\left(a_{i j}\right)$ be a fuzzy matrix of order 2 . Let $f: F_{2 \times 2} \rightarrow F[0,1]$ be the multiplicative functional such that the matrix A can be partitioned into two fuzzy matrices of order 2 then

$$
f\left(A^{K}\right)=f\left(A^{(1)}(i, j)\right)+f\left(A^{(2)}(i, j)\right)+f\left({ }_{K} A\right)
$$

Proof. Let $A=\left(a_{i j}\right)$ be a fuzzy matrix of order 2. The matrix A can be partitioned into two fuzzy matrices $A^{(1)}(i, j)$ and $A^{(2)}(i, j)$ where $A^{(1)}(i, j)$ be the fuzzy matrix whose elements are in the first column only and $A^{(2)}(i, j)$ be the fuzzy matrix having elements in the second column only.

Let, $\left(\begin{array}{ll}a_{11} & a_{12} \\ a_{21} & a_{22}\end{array}\right)=\left(\begin{array}{ll}a_{11} & 0 \\ a_{21} & 0\end{array}\right)+\left(\begin{array}{ll}0 & a_{12} \\ 0 & a_{22}\end{array}\right)$

$\left.A=A^{(1)}(i, j)\right)+A^{(2)}(i, j)$

But $A^{K}=A+\left({ }_{K} A\right)$

Therefore $f\left(A^{K}\right)=f\left(A+\left({ }_{K} A\right)\right)$

$=f\left(A^{(1)}(i, j)+f\left(A^{(2)}(i, j)\right)+f\left({ }_{K} A\right)\right)$

$f\left(A^{K}\right)=f\left(A^{(1)}(i, j)+f\left(A^{(2)}(i, j)\right)+f\left({ }_{K} A\right)\right)$

\section{References}

[1] H. Hashimoto, Convergence of powers of a Fuzzy Transitive matrix, Fuzzy Sets and Systems, 9(1983), 153-160.

[2] R. Hemasinha, N.R. Pal and J.C. Bezdek, The determinant of a fuzzy matrix with respect to $t$ and co-t norms, Fuzzy Sets and Systems, 87(1997), 297-306.

[3] A. Kandel, A Fuzzy Mathematical Techniques with Applications, Addison-Wesley, Tokyo, 1986.

[4] J.B. Kim, Determinant theory for Fuzzy Matrices, Fuzzy Sets and Systems, 29(1989), 349-356.

[5] K.H. Kim and F.W. Roush, Generalized fuzzy matrices, Fuzzy Sets and Systems, 4(1980), 293-315.

[6] J.B.Kim, Determinant theory for fuzzy and boolean matrices, Congressus Numerantium, 1988, 273-276.

[7] W. Kolodziejezyk, Convergence of powers of s-transitive fuzzy matrices, Fuzzy Sets and Systems, 26(1988), 127130.

[8] M.Z. Ragab and E.G. Emam, The determinant and adjoint of a square fuzzy matrix, Fuzzy Sets and Systems, 61(1994), 297-307.

[9] M.Z. Ragab and E.G. E. Emam, The Determinant and Adjoint of a square Fuzzy Matrix, Information Sciences, 84(1995), 209-220.

[10] M.G. Thomason, Convergence of powers of a fuzzy transitive matrix, Fuzzy Sets and Systems, 9(1983), 153-160.

[11] A.K. Shymal and M. Pal, Triangular fuzzy matrices, Iranian Journal of Fuzzy Systems, 4(2007), 75-87.

${ }^{\text {[12] }}$ L.A. Zadeh, Fuzzy Sets, Information and Control, 8(1965), 338-353.
$\operatorname{ISSN}(\mathrm{P}): 2319-3786$

Malaya Journal of Matematik

ISSN(O):2321 - 5666 